\title{
Mechanical Alteration Associated With Chemotherapeutic Resistance of Breast Cancer Cells
}

\author{
Soyeun Park \\ College of Pharmacy, Keimyung University, Daegu, Korea
}

\begin{abstract}
Background: The mechanical deformability of cancer cells has attracted particular attention as an emerging biomarker for the prediction of anti-cancer drug sensitivity. Nevertheless, it has not been possible to establish a general rubric for the identification of drug susceptibility in breast cancer cells from a mechanical perspective. In the present study, we investigated the mechanical alteration associated with resistance to adjuvant therapy in breast cancer cells.

Methods: We performed an 'atomic force microscopy (AFM)-based nanomechanical study' on 'drug-sensitive (MCF-7)' and 'drug-resistant (MCF-7/ADR)' breast cancer cells. We also conducted cell viability tests to evaluate the difference in doxorubicin responsiveness between two breast cancer cell lines. We carried out a wound closure experiment to investigate the motility changes associated with chemotherapeutic resistance. To elucidate the changes in molecular alteration that accompany chemotherapeutic resistance, we investigated the expression of vinculin and integrin-linked kinase-1-which are proteins involved in substrate adhesion and the actin cytoskeleton-using Western blotting analysis.

Results: A MTT assay confirmed that the dose-dependent efficacy of doxorubicin was reduced in MCF-7/ADR cells compared to that in MCF-7 cells. The wound assay revealed enhanced two-dimensional motility in the MCF-7/ADR cells. The AFM mechanical assay showed evidence that the drug-resistant breast cancer cells exhibited a significant decrease in mechanical deformability compared to their drug-sensitive counterparts. The mechanical alteration in the MCF-7/ADR cells was accompanied by upregulated vinculin expression. Conclusions: The obtained results manifestly showed that the altered mechanical signatures-including mechanical deformability and motility-were closely related with drug resistance in the breast cancer cells. We believe that this investigation has improved our understanding of the chemotherapeutic susceptibility of breast cancer cells.
\end{abstract}

(J Cancer Prev 2018;23:87-92)

Key Words: Elastic modulus, Atomic force microscopy, Breast neoplasms, Multi-drug resistance, Mechanobiology

\section{INTRODUCTION}

Breast cancer is the most prevalent cancer among women. ${ }^{1}$ Despite recent progress in the early screening of breast cancer, about $20 \%$ to $30 \%$ of breast cancer patients experience recurrence and metastatic progression. ${ }^{2}$ The median survival time of relapsed breast cancer patients varies from 12 to 24 months. ${ }^{3}$ Only $20 \%$ of relapsed patients survive 5 years. The standard adjuvant chemotherapy regimen to treat breast cancer involves doxorubicin. ${ }^{4}$ However, doxorubicin treatment has a very low response rate (25\% to $40 \%$ ) owing to acquired drug resistance. ${ }^{3}$ Chemotherapeutic resistance remains a serious obstacle to alleviating the menace of breast cancer. However, there is a lack of information on why cancer cells do not respond to chemotherapy, and thus acquire chemotherapeutic resistance.

Many mechanisms have been suggested as being responsible for the acquisition of drug resistance in breast cancer cells. These mechanisms include alterations in drug transport, metabolism, and drug targets, enhanced DNA repair, drug-detoxifying mechanisms, and dysregulation of the apoptotic pathway. ${ }^{5-7}$

Received May 27, 2018, Revised June 20, 2018, Accepted June 20, 2018

Correspondence to: Soyeun Park

College of Pharmacy, Keimyung University, 1095 Dalgubeol-daero, Dalseo-gu, Daegu 42601, Korea

Tel: +82-53-580-5164, Fax: +82-53-580-6654, E-mail: sypark20@kmu.ac.kr, ORCID: Soyeun Park, https://orcid.org/0000-0003-3989-9079

Copyright (C) 2018 Korean Society of Cancer Prevention

(c) This is an Open Access article distributed under the terms of the Creative Commons Attribution Non-Commercial License (http://creativecommons.org/icenses/by-nc/4.0) which permits unrestricted non-commercial use, distribution, and reproduction in any medium, provided the original work is properly cited. 
However, the mechanical alterations accompanying drug resistance have not been fully considered until recently.

Over the past decade, diverse experimental interventionsincluding 'micropipette aspiration', 'microplate stretcher', 'magnetic twisting cytometry', and 'optical stretchers'-have been devised to investigate the mechanical properties of biological cells. ${ }^{8}$ Among these, atomic force microscopy (AFM) has been the most utilized technique for nanomechanical investigations. AFM has several advantages including their adaptability to liquid environments, sub-nanonewton sensitivity, and nanometerscale lateral resolution. The most striking discovery made in biomechanical studies is that 'cancer cells are mechanically more compliant than normal cells', ${ }^{9-11}$ The enhanced mechanical deformability is a promising diagnostic marker for cancer. Furthermore, some chemotherapeutic agents induce the mechanical stiffening of ovarian cancer and leukemia. ${ }^{8,12,13}$ These agents include cisplatin, taxol, vincristine, and vinblastine. It has also been reported that the vascular complications found in leukemia patients after chemotherapy are closely correlated with enhanced cell stiffness and adhesion. ${ }^{14}$ However, there have been few investigations of the mechanical significance of chemotherapeutic resistance in breast cancer cells.

We also investigated whether the mechanical alteration associated with chemotherapeutic resistance accompanies the altered expression of the proteins associated with cell-matrix adhesion clusters, such as vinculin and integrin-linked kinase-1 (ILK-1). Vinculin is a structural protein found in focal adhesions, and is responsible for the maturation process of focal adhesions. ILK-1 transduces mechanical signals from focal adhesions to intracellular signaling pathways. It affects important cellular functions such as motility, adhesion, and spreading. Actin stress fibers tend to increase mechanical stiffness of cells and were nucleated from the focal adhesions. We hypothesized that the focal adhesion associated molecules including vinculin and ILK-1 might be correlated with the mechanical alterations in chemotherapeutic resistance.

In the present study, we investigated whether the drug resistance of breast cancer cells is accompanied by alterations to their mechanical deformability and motile behavior. To determine which molecular components mediate these mechanical alterations, we investigated the expression levels of vinculin and ILK-1 using western blotting analysis.

\section{MATERIALS AND METHODS}

\section{Cell culture}

We used 'drug-sensitive (MCF-7)' and 'drug-resistant (MCF-7/ADR)' breast cancer cells as model systems. The MCF-7 cells were supplied by the Korean Cell Line Bank (Seoul, Korea) and the MCF-7/ADR cells were kindly provided by Dr. M.K. Lee at Woosuk University, Korea. DMEM with $2 \mathrm{mM}$ L-glutamine was used to culture the cells at $37^{\circ} \mathrm{C}$ in $5 \% \mathrm{CO}_{2}$. We also included $10 \%$ FBS and $1 \%$ penicillin/streptomycin in the culture medium.

\section{Cell viability assay}

We carried out a MTT assay to evaluate the cytotoxic efficacy of doxorubicin. The cells were seeded with $200 \mu \mathrm{L}$ of the culture medium in a 96-well plate (approximately 3,000 cells per well), and were incubated overnight to achieve full attachment. The next day, doxorubicin $(0,10,50,100,500,1,000$, and 10,000 nM) was added to the wells. The cells were then incubated continuously at $37^{\circ} \mathrm{C}$ for 72 hours. We accessed cell viability using a CellTiter 96 AQueous One Solution Cell Proliferation Assay kit (Promega, Madison, WI, USA). After treatment with doxorubicin, the MTT reagent $(0.5 \mathrm{mg} / \mathrm{mL})$ was added to the wells. The 4 hours of additional incubation was granted at $37^{\circ} \mathrm{C}$ before taking the optical measurements. We used a Tecan Infinite F200 PRO plate reader (Tecan Trading AG, Männedorf, Switzerland) for the absorbance at $565 \mathrm{~nm}$. Absorbance relative to the absorbance of cells incubated in dimethyl sulfoxide alone was calculated to confirm cell viability.

\section{Wound closure assay}

We carried out a wound closure assay to determine the motile behavior of the cells. We seeded $1 \times 10^{6}$ cells into each well of a six-well plate, and incubated them in the growth medium to $90 \%$ confluence. A straight gap wound was created in each well using a sterilized micropipette tip. Fresh culture medium without FBS was provided for further incubation. Time-lapsed optical images were obtained at 0,12 , and 24 hours after scratching the cell layer.

\section{Atomic force microscopy assay}

A commercially available AFM (MFP3D ${ }^{\circledR}$; Asylum Research, Santa Barbara, CA, USA) was used for the measurements. We used a triangular cantilever with a round tip composed of a polystyrene sphere. We chose a round AFM tip because such tips have a well-defined contact area and produce relatively little stress unlike sharp tips. ${ }^{15}$ We used the thermal noise fluctuations method' to calibrate the spring constants of the cantilevers. 
Typical spring constants were measured as $0.01 \mathrm{~N} / \mathrm{m}$. Forcedistance $(f-d)$ curves were measured at a region near the cell center selected using the real-time images from an inverted microscope (IX-81 ${ }^{\circledR}$; Olympus, Tokyo, Japan) mounted on the AFM. The $f$ - $d$ curves were gathered within 1 second with a trigger force of 1 to $3 \mathrm{nN}$. We ensured a linear correlation between the force $(f)$ and the distance $(d)$ by acquiring an $f$ - $d$ curve on a hard substrate in advance. The physiological conditions were maintained using a BioHeater ${ }^{\mathrm{TM}}$ operated at $37^{\circ} \mathrm{C}$ and a fluid cell to provide the growth medium during the meaurements. ${ }^{16} \mathrm{We}$ stopped taking measurements 3 hours after the initial measurements to ensure normal cell function. Approximately $1 \times 10^{4}$ cells were cultured on a glass slide 2 days before the AFM measurements were made.

\section{Calculation of elastic constants}

We determined the elastic moduli from $f$ - $d$ curves. First, we converted the $f-d$ curves to force-indentation ( $f-\delta$ ) curves. The force applied to a cell was calculated as a multiplication of the spring constant $\kappa$ by the cantilever deflection. We then adapted the Hertz analysis shown in Eq. 1 to calculate the elastic moduli from the $f-\delta$ curves. According to Eq. 1, the elastic constant $K=$ $E /\left(1-V^{2}\right)$ hardly changes as the dimensionless quantity $\delta / R$ increases, because cells behave as homogeneous mechanical bodies within the indentation range. Here, $v$ and $R$ represent the Poisson ratio and the radius of the tip, respectively.

$$
K=\frac{E}{1-V^{2}} \frac{3}{4} \frac{f}{\sqrt{R \delta^{3}}}
$$

\section{Western blotting}

We extracted cell lysates from harvested cells. The protein concentration was quantified using a Protein Assay kit (Bio-Rad, Hercules, CA, USA). Electrophoresis was performed using cell lysates containing $40 \mu \mathrm{g}$ of total protein through polyacrylamide gels $(10 \%)$. The electrophoresed samples were transferred to a polyvinylidene difluoride membrane (Millipore, Billerica, MA, USA) for the identification of selected proteins. The blots were detected with anti-glyceraldehyde 3-phosphate dehydrogenase (anti-GAPDH), anti-vinculin, and anti-ILK-1 primary antibodies bought from Cell Signaling Technology (Danvers, MA, USA) or Abcam (Cambridge, UK). The corresponding HRP-conjugated secondary antibodies (Santa Cruz Biotechnology, Dallas, TX, USA) were applied and developed by an enhanced chemiluminescence reagent. We used a commercial image processing program (Image $)^{\circledR}$;
https://imagej.net/Welcome) to quantitatively evaluate the expression levels of vinculin and ILK-1. GAPDH served as a control.

\section{Statistical significance}

The obtained data are displayed as mean \pm SEM. To test statistical significance, we performed a Student's $t$-test or oneway analysis of variance on the differences among treated pairs.

\section{RESULTS}

\section{Doxorubicin resistance revealed by the MTT assay}

We assessed cell viability after exposing breast cancer cells to doxorubicin at various concentrations from 0 to $10,000 \mathrm{nM}$ for 72 hours. The MTT assay confirmed the dose-dependent cytotoxic effect of doxorubicin in both breast cancer cell lines. Furthermore, the increase in the cytotoxic dose indicated a decrease in doxorubicin susceptibility in the MCF-7/ADR cells (Fig. 1).

\section{Enhanced motility in drug-resistant breast cancer cells}

We carried out a wound healing assay to compare motility between the MCF-7 and MCF-7/ADR cells. Straight line gaps were generated by scratching cells grown on 6-well plates, and wound closure was monitored at 0,12 , and 24 hours after wound creation by taking bright-field images. Typical bright-field images are shown in Figure 2. Although there was a wider wound in the MCF-7 cells, the wound gap in the MCF-7/ADR cells had mostly closed 24 hours after the initial scratch was created. This

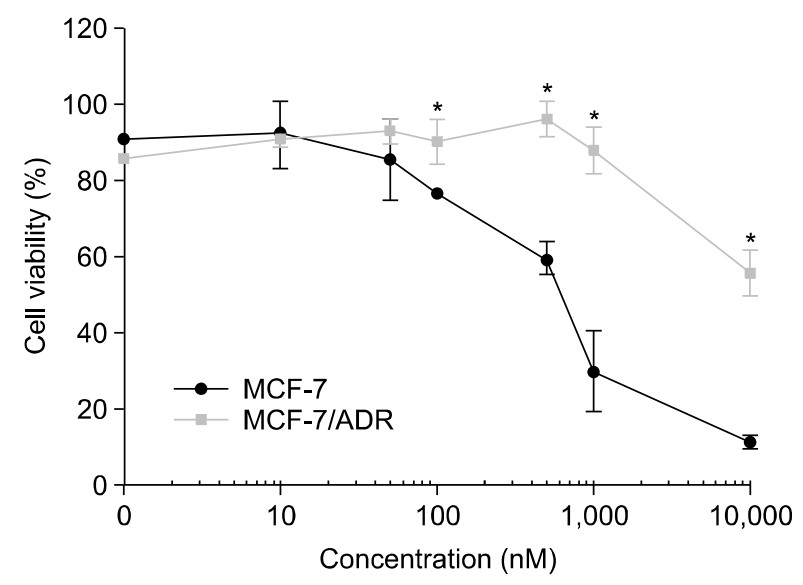

Figure 1. Effect of doxorubicin on breast cancer cells' viability. MTT assay was carried out to monitor cell viability with doxorubicin treatment for 3 days. Data are displayed as mean \pm SE $(n=4)$. $* P<0.01$. 


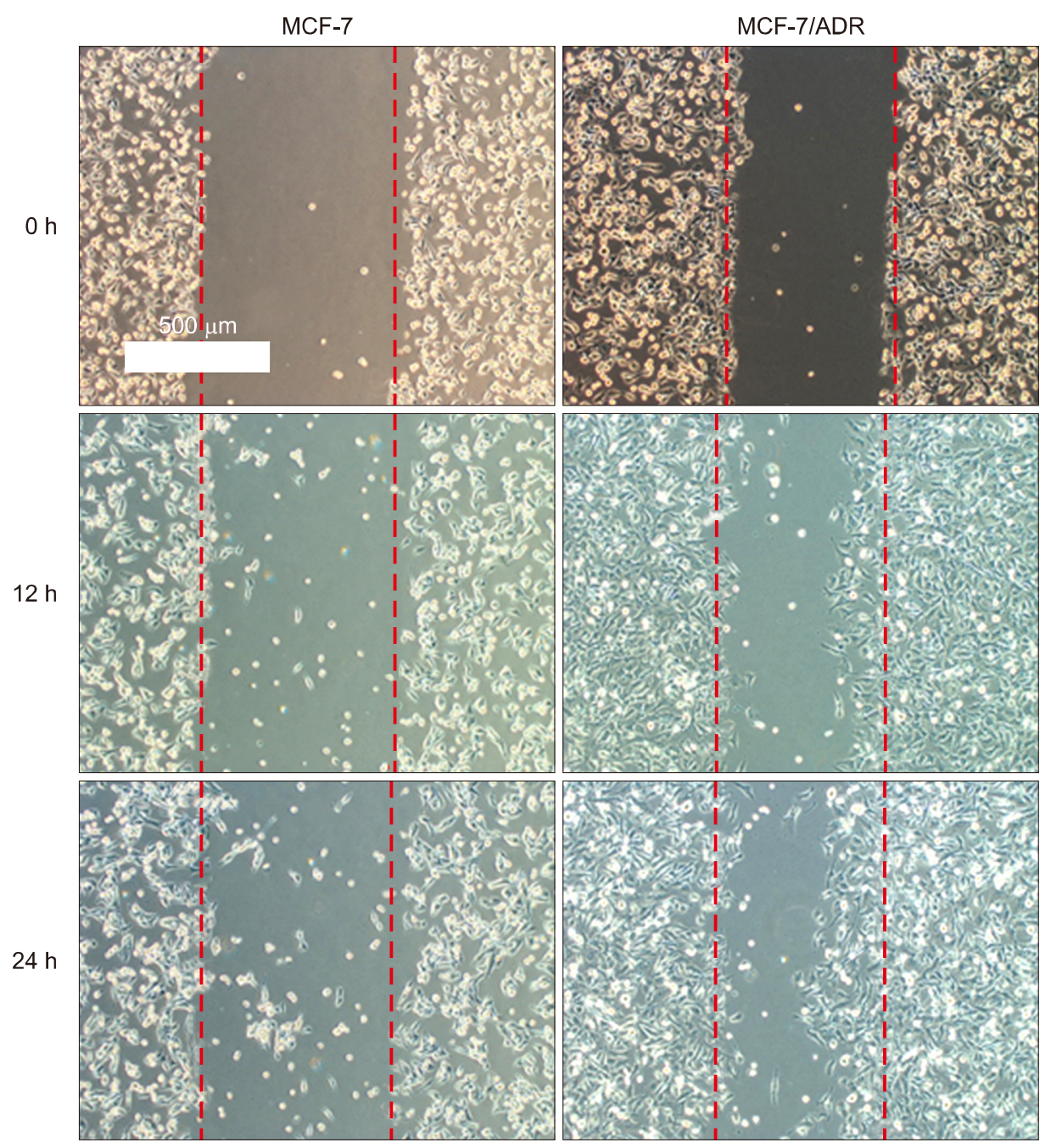

Figure 2. The results of the motility assay were confirmed by wound closure experiments. (A) Phase contrast images of wound closure were obtained every 12 hours after the wounds had been made. Faster closure of the wound gaps indicated that the MCF-7/ADR cells were significantly more motile than the MCF-7 cells.

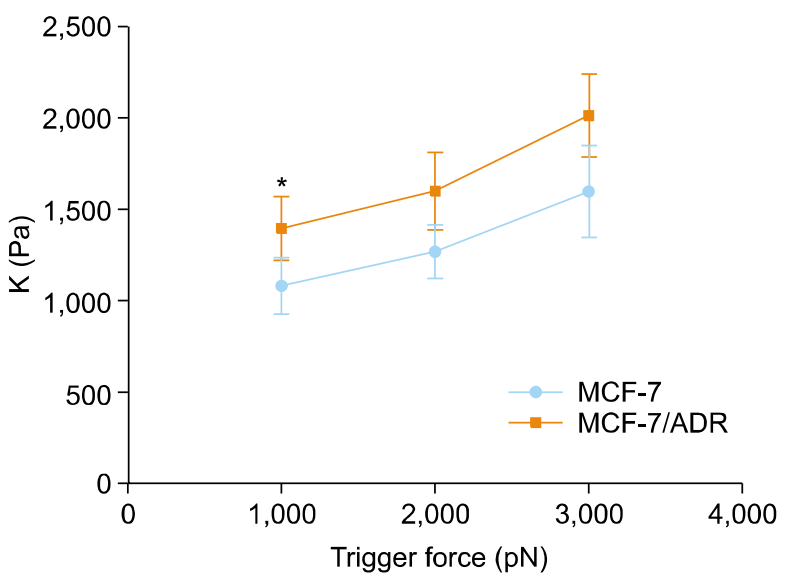

Figure 3. Differences in the elastic constant $(K)$ between the MCF-7 and MCF-7/ADR cells. The elastic constants were determined from the atomic force microscopy force-distance curves obtained by varying the trigger force from 1,000 to $3,000 \mathrm{pN}$. Over the observed trigger force range, the MCF-7/ADR cells $(\mathrm{n}=20)$ were mechanically stiffer than the MCF-7 $(\mathrm{n}=20)$ cells. $* P<0.05$. observation revealed that the MCF-7/ADR cells migrated much more than the MCF-7 cells.

\section{Mechanical hardening in drug resistance}

The AFM experiments were conducted to determine whether biomechanical alteration is a typical feature of acquired drug resistance in breast cancer. We considered the elastic constants $(K)$, which were successfully quantified by the conventional Hertz model from the obtained $f-\delta$ curves. MCF-7 cells exhibited larger deformation than MCF-7/ADR cells at the same trigger force. This result indicates an increase in the mechanical deformability of MCF-7 cells. The mechanical deformability of the observed breast cancer cells is displayed as mean \pm SEM in Figure 3. For both breast cancer cell lines, we observed a linear increase in the elastic constant as the applied trigger force increased from 1,000 to 3,000 $\mathrm{pN}$. Within the force range, the average elastic constants (mean \pm SEM) were 1,351 $\pm 124 \mathrm{~Pa}$ and 1,670 $\pm 137 \mathrm{~Pa}$ for the MCF-7 and 
MCF-7/ADR cells, respectively. We examined 20 cells per cell line for the statistical analysis. When we performed a Student's $t$-test on the elastic constants determined at the same trigger force, we found a significant difference in mechanical deformability between the drug-sensitive and -resistant cells $(P<0.05)$.

\section{Upregulated vinculin in drug-resistant cells}

Western blotting revealed that vinculin expression was upregulated two-fold in MCF-7/ADR compared to that in MCF-7 ( $P<$ $0.01)$ (Fig. 4). However, difference in the expression level of ILK-1 between the two breast cancer cell lines was negligible. Vinculin and ILK-1 are involved in the maturation process of cell-matrix adhesion. Whereas vinculin mainly acts as a linker protein between the integrin and actin filaments at larger focal adhesions, ILK-1 is associated with the cytoplasmic domain of $\beta_{r}$-integrin at smaller focal complexes, which subsequently mature further. Both proteins contribute to cytoskeletal reorganization, motility, and adhesion.

\section{DISCUSSION}

We accessed variations in the elastic constants of breast cancer cells depending on the drug susceptibility by AFM-based nanoindentation experiments. Within the force range of 1,000 to $3,000 \mathrm{pN}$, the drug-resistant breast cancer cells (MCF-7/ADR) were $30 \%$ less mechanically deformable than the drug-sensitive breast cancer cells (MCF-7), according to the obtained elastic constants (Fig. 3). The elastic constants of the MCF-7 cells were similar to those reported previously, indicating the validity of the AFM experiments. ${ }^{16}$ More importantly, the increased mechanical stiffness associated with doxorubicin resistance in the breast cancer cells resembled the significant mechanical deformability in cisplatin-resistant ovarian cancer cells. ${ }^{13,17}$ There is evidence that cisplatin-resistant ovarian cancer cells are mechanically stiffer than their cisplatin-sensitive counterparts. ${ }^{13,17,18}$

It has been postulated that the increased mechanical stiffness of drug-resistant cancer cells is closely correlated to an extensive reorganization of actin stress fibers. It is necessary to determine whether the observed mechanical reinforcement is associated with the maturation of focal adhesions, where the cells attach to the extracellular matrix. Fully mature focal adhesions-which are clusters of integrin and associated proteins-nucleate actin filaments, form more stress fibers, and thus contribute to the mechanical reinforcement of cells. ${ }^{19}$ Vinculin is a linkage protein that is associated with focal adhesions and accounts for the growth of integrin clusters. The loss of vinculin hinders cellsubstrate adhesion, cell migration, and the formation of lamellipodial extensions. We found that the drug-resistant breast cancer cells expressed twice as much vinculin as the drug-sensitive cells (Fig. 4). This upregulation of vinculin explains why the doxorubicin-resistant breast cancer cells were less mechanically deformable than the sensitive cells. Abundant vinculin might stimulate the formation of more actin stress fibers and thus contribute to an increase in mechanical stiffness in drug-resistant breast cancer cells. However, there was no significant difference between the cell lines in the expression of ILK-1, a protein that is abundant in tiny focal complexes, which grow into large focal adhesions.

This molecular signature also supports the increase in motile behavior of the MCF-7/ADR cells revealed by faster wound closure (Fig. 2). Vinculin supports the maturation of focal adhesions. The successive activation of Rho and Rho-associated kinase (ROCK) signaling and the myosin motor results in enhanced motility. ${ }^{20,21}$ We believe that the upregulation of vinculin contributes to the increase in cellular motility and mechanical stiffness.

It is evident that nanomechanical reinforcement and
A

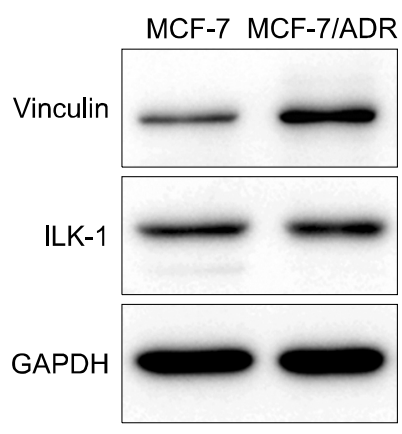

B

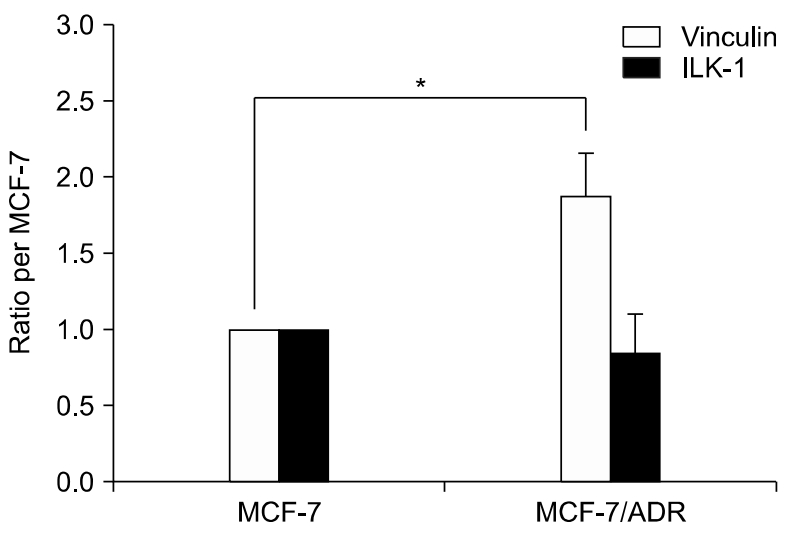

Figure 4. Differences in the expression levels of vinculin and integrin-linked kinase-1 (ILK-1) between the MCF-7 and MCR-7/ADR cells. (A) Representative western blotting images of vinculin and ILK-1 proteins. (B) Quantitative evaluation of vinculin and ILK-1 expression levels in the MCF-7/ADR cells normalized to those in the MCF-7 cells. GAPDH, glyceraldehyde 3-phosphate dehydrogenase. $* P<0.01$. 
enhanced motility are common characteristics of drug-resistant phenotypes of both breast and ovarian cancer cells. However, it is interesting that cisplatin-resistant ovarian cancer cells exhibit downregulated vinculin expression, indicating inconsistency between breast and ovarian cancers. ${ }^{17}$ We postulated that this discrepancy might be correlated with the fact that ovarian cancer cells exhibit very low values of mechanical stiffness. ${ }^{16}$ When the mechanical stiffness is very low as in ovarian cancer, the upregulated vinculin might not be fully activated, not be able to be incorporated into focal adhesions, and thus contribute to mechanical strength of cells. However, a more sophisticated investigation is required to resolve this issue.

In conclusion, we found substantial changes in mechanical deformability and motility associated with doxorubicin-resistant breast cancer cells. As a molecular signature, upregulated vinculin expression might be responsible for such alterations. We believe that our AFM-based nanomechanical study will provide novel insights into how cancer cells are desensitized to chemotherapeutic treatments.

\section{ACKNOWLEDGMENTS}

This study was supported by a grant from the Dongil Culture \& Scholarship Foundation in 2018.

The author thanks Dr. M.K. Lee of Woosuk University for graciously providing MCF-7/ADR cells.

\section{CONFLICTS OF INTEREST}

No potential conflicts of interest were disclosed.

\section{REFERENCES}

1. Jemal A, Bray F, Center MM, Ferlay J, Ward E, Forman D. Global cancer statistics. CA Cancer J Clin 2011;61:69-90.

2. Berman AT, Thukral AD, Hwang WT, Solin LJ, Vapiwala N. Incidence and patterns of distant metastases for patients with early-stage breast cancer after breast conservation treatment. Clin Breast Cancer 2013;13:88-94.

3. Gonzalez-Angulo AM, Morales-Vasquez F, Hortobagyi GN. Overview of resistance to systemic therapy in patients with breast cancer. Adv Exp Med Biol 2007;608:1-22.

4. Henderson IC, Berry DA, Demetri GD, Cirrincione CT, Goldstein LJ, Martino S, et al. Improved outcomes from adding sequential
Paclitaxel but not from escalating Doxorubicin dose in an adjuvant chemotherapy regimen for patients with node-positive primary breast cancer. J Clin Oncol 2003;21:976-83.

5. Sun YL, Patel A, Kumar P, Chen ZS. Role of ABC transporters in cancer chemotherapy. Chin J Cancer 2012;31:51-7.

6. Mukohara T. Mechanisms of resistance to anti-human epidermal growth factor receptor 2 agents in breast cancer. Cancer Sci 2011;102:1-8.

7. Wong RS. Apoptosis in cancer: from pathogenesis to treatment. J Exp Clin Cancer Res 2011;30:87.

8. Suresh S. Biomechanics and biophysics of cancer cells. Acta Biomater 2007:3:413-38.

9. Lekka M, Pogoda K, Gostek J, Klymenko O, Prauzner-Bechcicki S, Wiltowska-Zuber J, et al. Cancer cell recognition-mechanical phenotype. Micron 2012;43:1259-66.

10. Guck J, Schinkinger S, Lincoln B, Wottawah F, Ebert S, Romeyke $\mathrm{M}$, et al. Optical deformability as an inherent cell marker for testing malignant transformation and metastatic competence. Biophys J 2005;88:3689-98.

11. Ochalek T, Nordt FJ, Tullberg K, Burger MM. Correlation between cell deformability and metastatic potential in B16-F1 melanoma cell variants. Cancer Res 1988;48:5124-8.

12. Grzanka A, Grzanka D, Orlikowska M. Cytoskeletal reorganization during process of apoptosis induced by cytostatic drugs in K-562 and HL-60 leukemia cell lines. Biochem Pharmacol 2003;66:1611-7.

13. Sharma S, Santiskulvong C, Bentolila LA, Rao J, Dorigo O, Gimzewski JK. Correlative nanomechanical profiling with super-resolution F-actin imaging reveals novel insights into mechanisms of cisplatin resistance in ovarian cancer cells. Nanomedicine 2012;8:757-66.

14. Lam WA, Rosenbluth MJ, Fletcher DA. Chemotherapy exposure increases leukemia cell stiffness. Blood 2007;109:3505-8.

15. Park S, Koch D, Cardenas R, Käs J, Shih CK. Cell motility and local viscoelasticity of fibroblasts. Biophys J 2005:89:4330-42.

16. Park S, Lee YJ. AFM-based dual nano-mechanical phenotypes for cancer metastasis. J Biol Phys 2014:40:413-9.

17. Seo YH, Jo YN, Oh YJ, Park S. Nano-mechanical reinforcement in drug-resistant ovarian cancer cells. Biol Pharm Bull 2015:38: 389-95.

18. Kapoor A, Barai A, Thakur B, Das A, Patwardhan SR, Monteiro M, et al. Soft drug-resistant ovarian cancer cells migrate via two distinct mechanisms utilizing myosin II-based contractility. Biochim Biophys Acta 2018;1865:392-405.

19. Ingber DE, Wang N, Stamenovic D. Tensegrity, cellular biophysics, and the mechanics of living systems. Rep Prog Phys 2014;77:046603.

20. Hanna S, El-Sibai M. Signaling networks of Rho GTPases in cell motility. Cell Signal 2013;25:1955-61.

21. Sahai E, Marshall CJ. Differing modes of tumour cell invasion have distinct requirements for Rho/ROCK signalling and extracellular proteolysis. Nat Cell Biol 2003;5:711-9. 